\title{
Heterogeneity and chronology of PTEN deletion and ERG fusion in prostate cancer
}

\author{
Antje Krohn ${ }^{1,4}$, Fabian Freudenthaler ${ }^{1,4}$, Silvia Harasimowicz ${ }^{1}$, Martina Kluth ${ }^{1}$, \\ Sarah Fuchs ${ }^{1}$, Lia Burkhardt ${ }^{1}$, Phillip Stahl ${ }^{1}$, Maria C Tsourlakis ${ }^{1}$, Melanie Bauer ${ }^{1}$, \\ Pierre Tennstedt ${ }^{2}$, Markus Graefen ${ }^{2}$, Stefan Steurer ${ }^{1}$, Hueseyin Sirma ${ }^{1}$, Guido Sauter ${ }^{1}$, \\ Thorsten Schlomm ${ }^{2,3}$, Ronald Simon ${ }^{1}$ and Sarah Minner ${ }^{1}$ \\ ${ }^{1}$ Institute of Pathology, University Medical Center Hamburg-Eppendorf, Hamburg, Germany; ${ }^{2}$ Martini-Clinic, \\ Prostate Cancer Center, University Medical Center Hamburg-Eppendorf, Hamburg, Germany and \\ ${ }^{3}$ Department of Urology, Section for Translational Prostate Cancer Research, University Medical Center \\ Hamburg-Eppendorf, Hamburg, Germany
}

\begin{abstract}
TMPRSS2:ERG fusions, in combination with deletion of the phosphatase and tensin homolog (PTEM) tumor suppressor, have been suggested to cooperatively drive tumor progression in prostate cancer. We utilized a novel heterogeneity tissue microarray containing samples from 10 different tumor blocks of 189 prostatectomy specimens to study heterogeneity of genomic PTEN alterations in individual foci. PTEN alterations were found in $48 / 123(39 \%)$ analyzable individual tumor foci, including 40 foci with deletions, 7 with deletion and rearrangement, and 1 focus with rearrangement only. PTEN was homogeneously aberrant in only $4(8 \%)$ and heterogeneously in $44(92 \%)$ of the foci. We found a specific sequence of molecular events from PTEN breakage followed by deletion of DNA sequences flanking the breakpoint, resulting in homozygous deletion. The observation that 16 of 19 foci with homogeneous ERG positivity had focal PTEN alterations but none of 10 foci with PTEN alterations had focal ERG positivity $(P<0.0001)$ suggests that $P T E N$ alterations typically develop subsequent to ERG fusions. We demonstrate a high level of intratumoral heterogeneity of PTEN alterations with deletions and rearrangements that challenges potential PTEN routine diagnosis testing in biopsies. The observation that PTEN alterations develop subsequent to ERG fusion strongly suggests that ERG expression may directly drive development of PTEN aberrations.

Modern Pathology (2014) 27, 1612-1620; doi:10.1038/modpathol.2014.70; published online 25 April 2014
\end{abstract}

Keywords: deletion; ERG; heterogeneity; prostate cancer; PTEN; translocation

Prostate cancer is the most frequent malignancy in males and responsible for $>250000$ deaths per year worldwide. ${ }^{1}$ These tumors typically show marked histological and molecular heterogeneity, which can be problematic if a potential biomarker or therapeutic target is found, as only minute core needle biopsies are available for the initial diagnosis. However, comparing the extension of multiple heterogeneous molecular alterations within one cancer can also serve to answer biological questions such as the sequel in which these changes occurred. A prerequisite for such an extensive

Correspondence: Dr S Minner, MD, Institute of Pathology, University Medical Center Hamburg-Eppendorf, Martinistr. 52, Hamburg 20246, Germany.

E-mail: s.minner@uke.de

${ }^{4}$ These authors contributed equally to this study.

Received 8 January 2014; revised 17 March 2014; accepted 18 March 2014; published online 25 April 2014 heterogeneity analysis is that the entire cancer bulk is assessable for molecular analysis. To facilitate such whole tumor analyses, we have previously developed a prostate cancer heterogeneity tissue microarray (tissue microarray) platform. ${ }^{2}$ This tissue microarray platform contains samples from 10 distant tumor areas each of 189 large prostate cancers, thus enabling a high-throughput mapping of molecular features across the entire tumors. Our initial analysis of this tissue microarray revealed that the prostate cancer-specific TMPRSS2:ERG fusions is heterogeneous in $>70 \%$ of $E R G$-positive prostate cancers. $^{2}$

Inactivation of the phosphatase and tensin homolog $(P T E N)$ gene by genomic deletion or rearrangement, including intragenic breakage and translocation, is another key molecular event in prostate cancer. PTEN deletion or rearrangement has been reported in $20-30 \%$ of prostate carcinomas, and is linked to particularly aggressive cancers. ${ }^{3-5}$ Mouse 
models of prostate cancer suggest that PTEN inactivation and $E R G$ fusion may cooperate in triggering development and progression of prostate cancer. ${ }^{6,7}$ Studies comparing PTEN and ERG fusion status have demonstrated that these alterations frequently coexist. ${ }^{3,4,8-12}$ PTEN deletions are about three times more frequent in fusion-positive prostate cancers than in fusion-negative cancers. ${ }^{10}$ The mechanisms explaining the frequent coexistence of PTEN deletions and TMPRSS2:ERG fusions in prostate cancer are unknown. In principle, it is possible that TMPRSS2:ERG fusions facilitate development of PTEN deletions, that PTEN deletions facilitate development of TMPRSS2:ERG fusions or that a specific molecular background facilitates development of both alterations simultaneously.

In order to distinguish between these possibilities, we extended our heterogeneity tissue microarray analysis for PTEN alterations, including deletions and breakage, and compared the PTEN and ERG status in the same tumor areas. The data from this project show high heterogeneity for PTEN aberrations in prostate cancer and suggest that presence of $E R G$ activation facilitates development of PTEN aberrations, while PTEN altered cancers do not show an increased risk for developing additional TMPRSS2:ERG fusions.

\section{Materials and methods}

\section{Patient Samples and Tissue Microarray Construction}

Usage of the TMA for this study has been approved by the local ethics committee (AZ: WF-049/09; $\$ 12$ HmbKHG). The prostate cancer heterogeneity tissue microarray utilized in this study has been described in detail elsewhere. ${ }^{13} \mathrm{~A}$ total of 189 formalin-fixed prostatectomy specimens with tumor in at least 10 different tissue blocks were selected for tissue microarray construction. For each cancer, the number of independent tumor foci was determined according to Wise et al. ${ }^{14}$ In brief, tumor areas were defined as part of a single focus if they were within $3 \mathrm{~mm}$ of each other in any section or within $4 \mathrm{~mm}$ on adjacent sections. This method identified 1-6 independent tumor foci in our prostate cancers. Seventy-six prostates had 1 tumor focus, 48 prostates had 2 tumor foci, 28 prostates had 3 tumor foci and 37 prostates had 4 or more tumor foci. The latter group also included nine prostates that contained multiple small and very small tumor foci rather than one or several clearly distinguishable tumor masses. One $0.6 \mathrm{~mm}$ tumor tissue core was removed from each of the 10 tumor blocks per patient. The 10 tissue cores were distributed across 10 different tissue microarray blocks, so that the complete heterogeneity tissue microarray consists of 10 tissue microarray blocks, each containing one tissue sample of all 189 patients. The localization of each arrayed tumor sample inside the prostate was recorded in order to distinguish between different tumor foci. Presence of cancer was histologically confirmed in each tissue spot. Normal prostate glands were immunohistochemically identified using the antibody 34BE12 (clone MA903, 1:12.5, pH7.8 DAKO, Glostrup, Denmark) for basal cell detection.

\section{Fluorescence In Situ Hybridization (FISH)}

FISH was used to detect genomic PTEN deletions and translocations. For deletion analysis, a dualcolor FISH probe was constructed from two Spectrum Orange-labeled BAC clones (RP11-380G05, RP11-813O03; Source Bioscience, Nottingham, UK) and a commercial Spectrum Green-labeled centromere 10 (CEP10) reference probe (Abbott Molecular, Wiesbaden, Germany). For PTEN translocation analysis, a dual-color FISH break-apart probe consisting of two Spectrum Orange-labeled BACs (5'PTEN: RP11-659F22, RP11-79A15) and two Spectrum Green-labeled BACs (3'PTEN: RP11-765C10, RP11-813O03) flanking the PTEN gene were used. The localization of all probes and representative FISH images are shown in Figures 1a and b, respectively. Freshly cut $4 \mu \mathrm{m}$ tissue microarray sections were deparaffinized and proteolytically pretreated using a commercial kit (paraffin pretreatment reagent kit; Abbott Molecular), followed by dehydration in 70, 80 and $96 \%$ ethanol, air-drying and denaturation for $10 \mathrm{~min}$ at $72{ }^{\circ} \mathrm{C}$ in $70 \%$ formamide2x SSC solution. Hybridization was done overnight at $37^{\circ} \mathrm{C}$ in a humidified chamber; slides were then washed and counterstained with $0.2 \mu \mathrm{mol} / \mathrm{l}$ 4'-6diamidino-2-phenylindole in an antifade solution.

\section{Scoring of FISH}

The stained slides were visually inspected under an epifluorescence microscope. At least 30 different tumor cell nuclei were inspected for the FISH signal numbers per tissue spot. PTEN deletion was defined as follows: homozygous PTEN deletion was assumed if PTEN deletion probe signals were completely lacking in $\geq 60 \%$ of tumor nuclei, while PTEN FISH signals were present in adjacent normal cells. Heterozygous deletion of PTEN was defined as presence of less PTEN signals than centromere 10 probe signals of $\geq 60 \%$ tumor nuclei. For structural PTEN rearrangement analysis using the break-apart probe, tumors were defined as 'normal' when two pairs of overlapping orange and green signals were seen per cell nucleus. A PTEN rearrangement was assumed if at least one split signal consisting of a separate orange and green signal was observed per cell nucleus in $\geq 60 \%$ of the tumor cell nuclei (indicating balanced translocations) or if individual orange and green signals from the overlapping orange/green signal were lost (indicating deletions with breakpoint inside the PTEN gene or 
a

\begin{tabular}{|c|c|c|c|c|c|c|c|}
\hline $89.30 \mathrm{Mb}$ & $89.40 \mathrm{Mb}$ & $89.50 \mathrm{Mb}$ & $89.60 \mathrm{Mb}$ & $89.70 \mathrm{Mb}$ & $89.80 \mathrm{Mb}$ & $89.90 \mathrm{Mb}$ & $90.00 \mathrm{Mb}$ \\
\hline \multicolumn{2}{|c|}{$q 23.2$} & \multicolumn{6}{|c|}{$q 23.31$} \\
\hline
\end{tabular}

\section{PTEN}

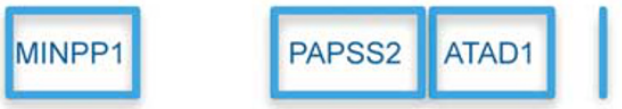

KLLN

\section{PTEN deletion probe}

\section{5' break apart probe}

\section{3' break apart probe}
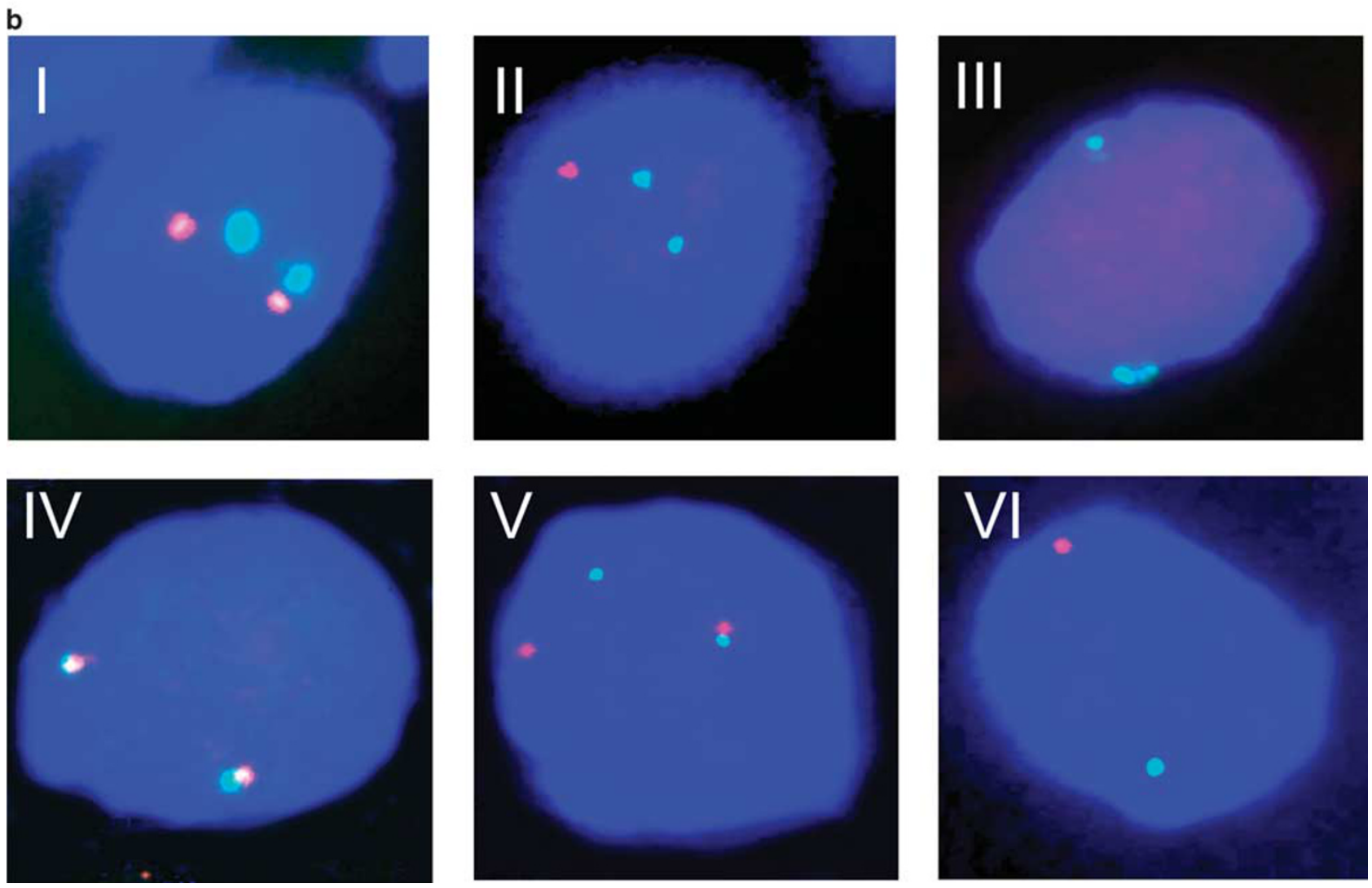

Figure 1 (a) Localization of the FISH probes used for detection PTEN deletion and intragenic breaks. For detection of PTEN deletion, a PTEN deletion probe covering the entire PTEN gene and $3^{\prime}$ adjacent DNA, and a spectrum green-labeled centromere 10 control probe (data not shown), were co-hybridized. For detection of PTEN breakage, two differentially labeled probes corresponding to the $5^{\prime}$ and $3^{\prime}$ edges of PTEN were co-hybridized. (b) Examples of PTEN aberrations as detected by FISH. (I-III) Examples of the deletion probe: (I) normal copy number with two red PTEN signals and two green centromere 10 signals, (II) heterozygous deletion of PTEN showing loss of one red signal, but two green centromere signals, (III) homozygous deletion of PTEN lacking PTEN signals but showing two green centromere 10 signals. (IV-VI) Examples of the break-apart probe: (IV) intact PTEN as indicated by presence of two adjacent red (3' PTEN) and green (5' PTEN) signals, (V) translocation of PTEN showing one normal, adjacent red/green signal and a split of the second one into clearly separated green and red signals, (VI) heterozygous deletion of one allele and translocation of the remaining allele.

imbalanced translocations). Presence of only one overlapping orange/green signal in $>60 \%$ of tumor cells was considered a heterozygous deletion.
Tumors with complete lack of overlapping orange/ green signals were regarded as homozygous deletions provided that FISH signals were present in 
adjacent normal cells. A final score was assigned to each tissue spot that was analyzable for both the PTEN deletion and the break-apart probe according to the following criteria: normal (no deletion and no rearrangement), heterozygous inactivation (heterozygous deletion or rearrangement of one allele), homozygous inactivation (homozygous deletion or heterozygous deletion combined with rearrangement of the remaining allele or rearrangement of both alleles). In case of polyploidy, presence of normal PTEN FISH signals and split signals was rated as heterozygous inactivation. A total of 103 tissue spots that had interpretable results with only one of the two probes were scored based on the respective FISH finding only.

\section{ERG Immunohistochemistry}

ERG immunohistochemistry data were available from a previous study. ${ }^{15}$

\section{Large Section Analysis}

To validate the PTEN tissue microarray findings, corresponding tumor blocks were selected from 15 tumor foci showing either a PTEN alteration in only one tissue core $(n=10)$ or in all but one tissue core $(n=5)$.

\section{Statistics}

Statistical calculations were performed using JMP 9.0 statistical software (SAS Institute, Cary, NC, USA). Contingency tables were calculated with the $\chi^{2}$ test to investigate the relationship between the degree of PTEN and ERG heterogeneity.

\section{Results}

\section{Technical Issues}

A total of 1890 tissue spots were included in this study. A total of 887 and 907 tissue spots were interpretable with the PTEN deletion probe and with the PTEN break-apart probe, respectively. As all tumors with deletions according to the deletion probe also showed FISH signal losses with the break-apart probe, the deletion status was assigned solely based on the result of the break-apart probe in 125 tissue spots lacking results with the deletion probe. In additional 103 tissue spots that had a result with the deletion probe but were not interpretable with the break-apart probe, the PTEN status was assigned based on the deletion probe only, and no breakage was assumed in these cases. This assumption was based on the low overall rate of PTEN breaks (1\%) in tumors without simultaneous deletion. ${ }^{16}$ Accordingly, the PTEN FISH status was available from 1008 (53\%) tissue spots. Reasons for analysis failure included missing tissue spots on the tissue microarray sections $(n=111)$, lack of unequivocal tumor cells in the tissue spots $(n=501)$ or insufficient hybridization for both FISH probe sets $(n=270)$. The 1008 interpretable tissue spots belonged to 173 different tumor foci obtained from 136 prostatectomy specimens. As different tumor foci within the same prostate represent individual cancers, and because some tumor foci had only few interpretable tissue spots, we refer to different tumor foci in our analysis (rather than to different patients) and excluded all foci with $<3$ interpretable tissue cores. In summary, 821 tissue spots originating from 123 tumor foci with at least three interpretable tissue spots obtained from 118 patients were included into all subsequent analyses.

\section{Heterogeneity of PTEN Alterations}

PTEN alterations (including deletion and rearrangement) were found in 48 (39\%) of the 123 individual tumor foci belonging to 47 patients. We observed a high degree of intratumoral heterogeneity: 44 (92\%) of the foci were heterogeneously, but only $4(8 \%)$ were homogeneously PTEN altered. In contrast, the inactivation status (heterozygous or homozygous) was usually identical in all PTEN altered tissues of the same focus, with homozygous inactivation being more frequent $(25 / 48,52 \%)$ than heterozygous inactivation $(17 / 48,35 \%)$. Only six (13\%) foci showed a mixture of tissue spots with homo- and heterozygous inactivation. All results are summarized in Figure 2.

\section{Sequel of PTEN Rearrangement and Deletion}

Of the 48 PTEN altered foci, 40 foci had deletions only, 7 foci had structural PTEN rearrangements in addition to deletions and 1 focus had a PTEN rearrangement as the sole detectable PTEN alteration. In the 47 foci with deletion and/or structural rearrangements, deletions and rearrangements of PTEN were strongly linked. Rearrangements were found in 7/47 tumor foci with PTEN deletion, but only in 1/76 foci with normal PTEN copy numbers $(P=0.003)$. The presence of tissue spots with both deletions and rearrangements of PTEN within the same cancer focus enabled us to study the sequel of PTEN breakage and deletion. Of these seven foci, six had $\geq 2$ interpretable tissue spots, some of which showed structural PTEN rearrangements, while others had partial and/or complete deletions of PTEN. This pattern of alterations is consistent with a specific sequel of molecular events starting with PTEN breakage and followed by deletion of DNA sequences flanking the breakpoint (Figure 3), including multiple $5^{\prime}$ adjacent genes (MINPP1, PAPSS2, $A T A D 1$ and $K L L N$ ) covered by our break-apart FISH probe (Figure 1). 


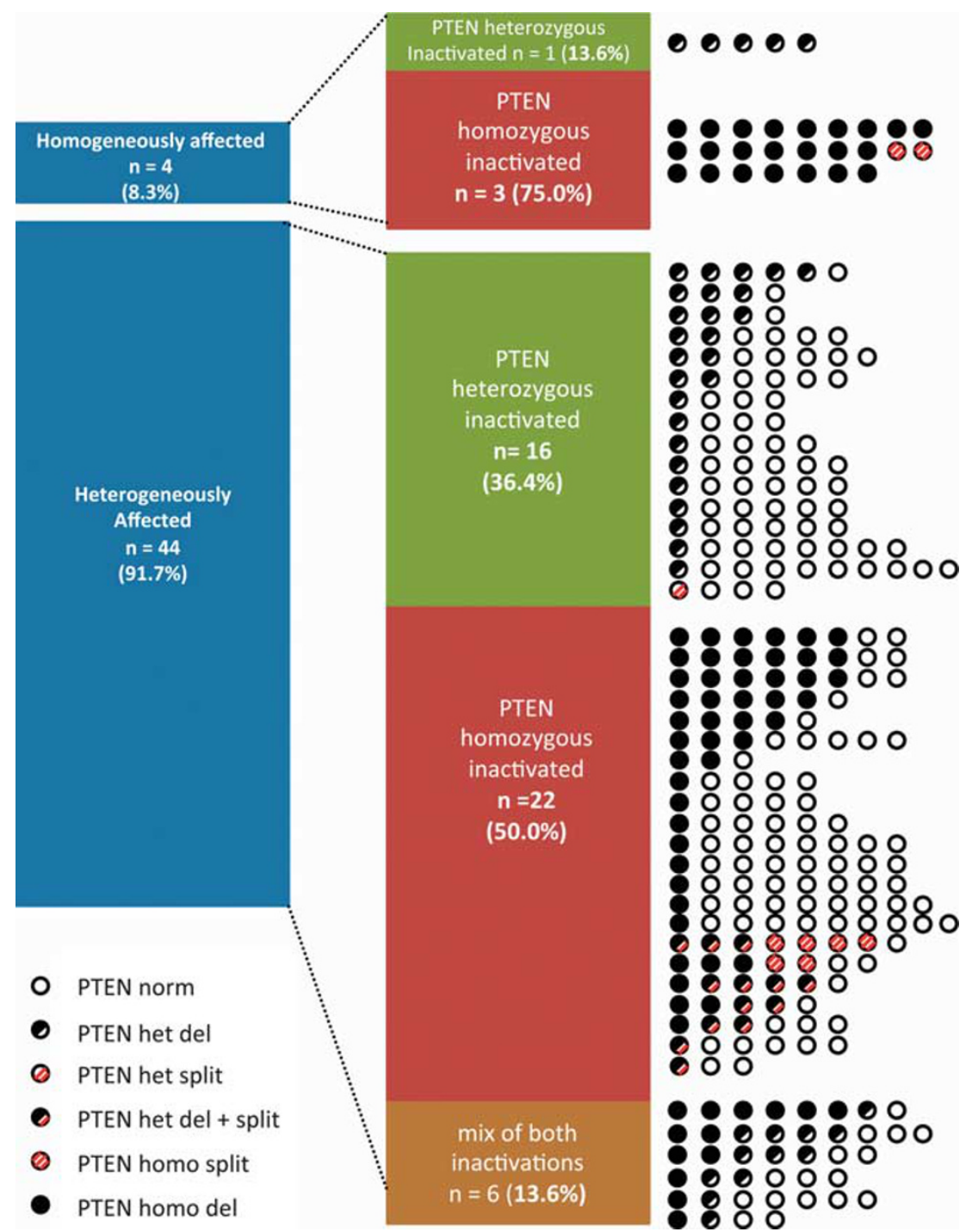

Figure 2 Summary of all PTEN FISH results in 48 tumor foci with PTEN alterations. 'Homozygously' and 'heterozygously affected' indicates foci with PTEN alterations in all analyzable tissue spots and foci with an admixture of tissue samples with and without PTEN alterations. Circles represent the individual tissue cores belonging to the same tumor foci. The number of interpretable tissue cores per foci ranged between 3 and 10.

\section{Association to $E R G$}

ERG data were available from a previous study in 786 of $821(96 \%)$ tissue spots with informative PTEN deletion and break-apart status. A positive ERG status was strongly linked to presence of PTEN alterations in these samples. PTEN aberrations were seen in $120(36 \%)$ of $332 E R G$ positive, but only in $26(6 \%)$ of $454 E R G$-negative tissue spots $(P<0.0001$; Figure 4).

\section{Sequel of PTEN Alterations and ERG Fusion}

To address the question whether alterations of $E R G$ and PTEN occur in a specific order, we searched for homogeneously ERG-positive foci with focal PTEN inactivation and large PTEN inactivated tumor areas containing focal $E R G$ positivity. Figure 5 shows the $E R G$ and PTEN findings in all 29 tumor foci that were suitable for such an analysis. Sixteen of 19 foci with homogenous ERG positivity had focal PTEN alterations but none of the 10 foci with homogeneous or heterogeneous PTEN alterations had focal $E R G$ positivity $(P<0.0001)$.

\section{Large Section Validation}

Large section analysis of 15 tumor foci with intrafocal PTEN heterogeneity confirmed presence of small areas with genomic PTEN alterations in large $E R G$-positive tumor foci. Heterogeneous findings occurred not only between different areas within one tumor focus but also within the area represented 


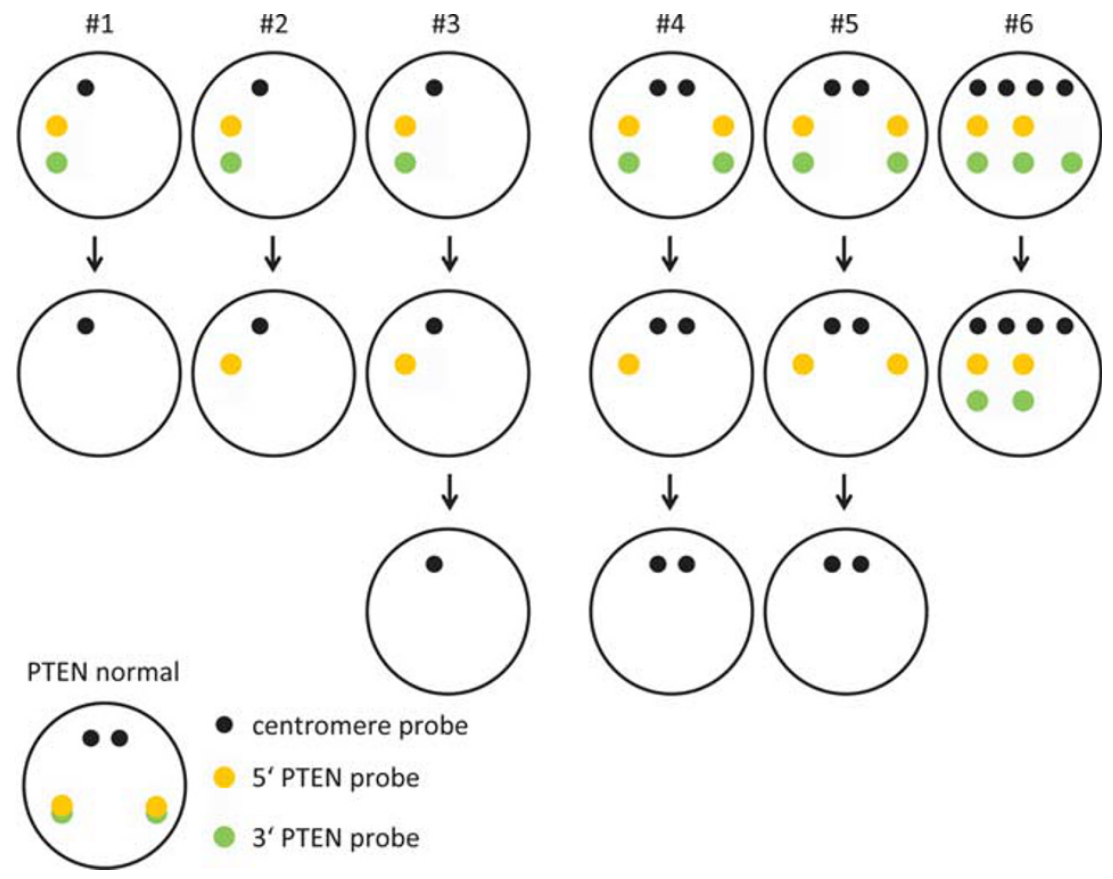

Figure 3 Schematic representation of the FISH findings in six cases with at least two tissue spots affected by PTEN rearrangements. Black dots correspond to the centromere 10 probe, yellow and green dots represent the corresponding PTEN break-apart FISH signals. All six cases showed translocations of all present alleles, subsequent loss of the break-apart signals mostly starting at the green $3^{\prime}$ sequence, followed by the orange $5^{\prime}$ sequence and finally loss of all FISH signals. For reference, the spot pattern of a normal case (no deletion, no rearrangement) is indicated in the bottom left corner.

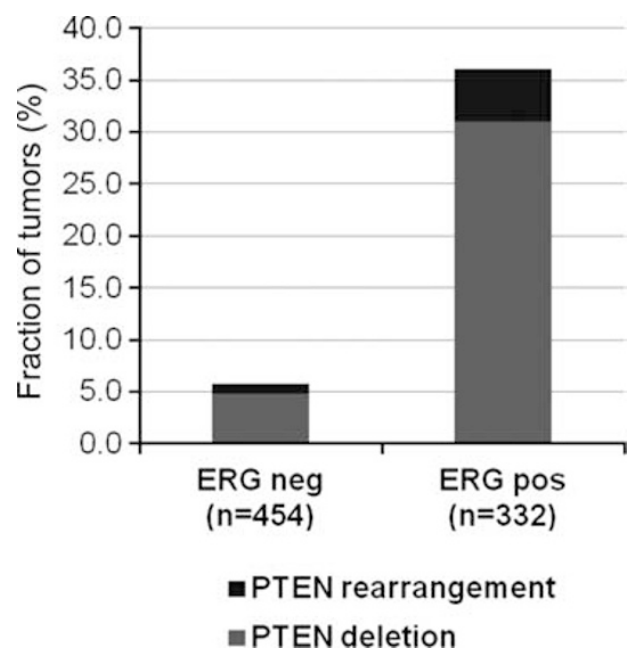

Figure 4 Association between immunohistochemical ERG expression and PTEN alterations evaluated in a spot by spot analysis.

by a single tissue block. Examples of two cases with heterogeneous FISH findings are shown in Figure 6.

\section{Discussion}

The results of our study show maximal heterogeneity for PTEN alterations in prostate cancer. Almost all $(92 \%)$ tumors with PTEN alterations also con-

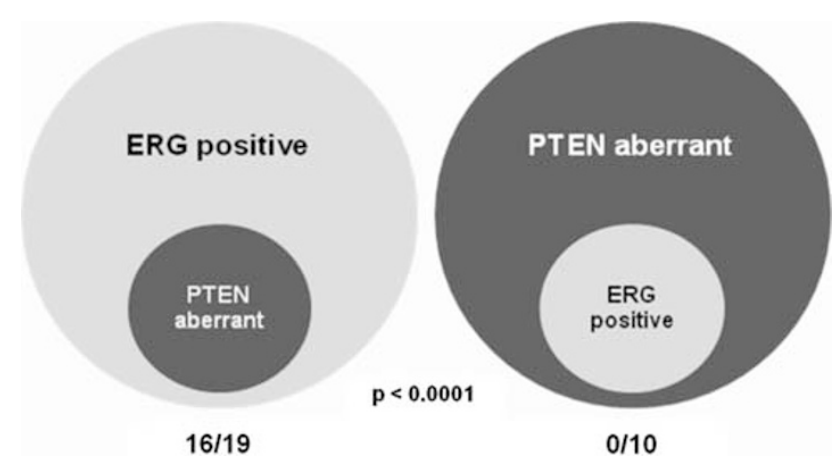

Figure 5 Frequent (16/19 foci) presence of focal areas of PTEN alterations in $E R G$-positive tumors but lack (0/10 foci) of focal areas of ERG positivity in PTEN altered tumors indicate that ERG fusion precedes PTEN alterations but not vice versa.

tained areas without PTEN alterations in the same tumor focus. In about half of the tumors, PTEN alterations were only found in one single tumor area, suggesting that PTEN alterations often occur in late stages of tumor progression. Large section analysis and validation of the tumor cell content in reference tissue microarray sections excluded technical errors or misinterpretation of individual tumor spots as a possible reason for artificial heterogeneity in cases with a single PTEN altered tumor area. Our findings are also supported by previous studies reporting heterogeneity of PTEN alterations, 
a
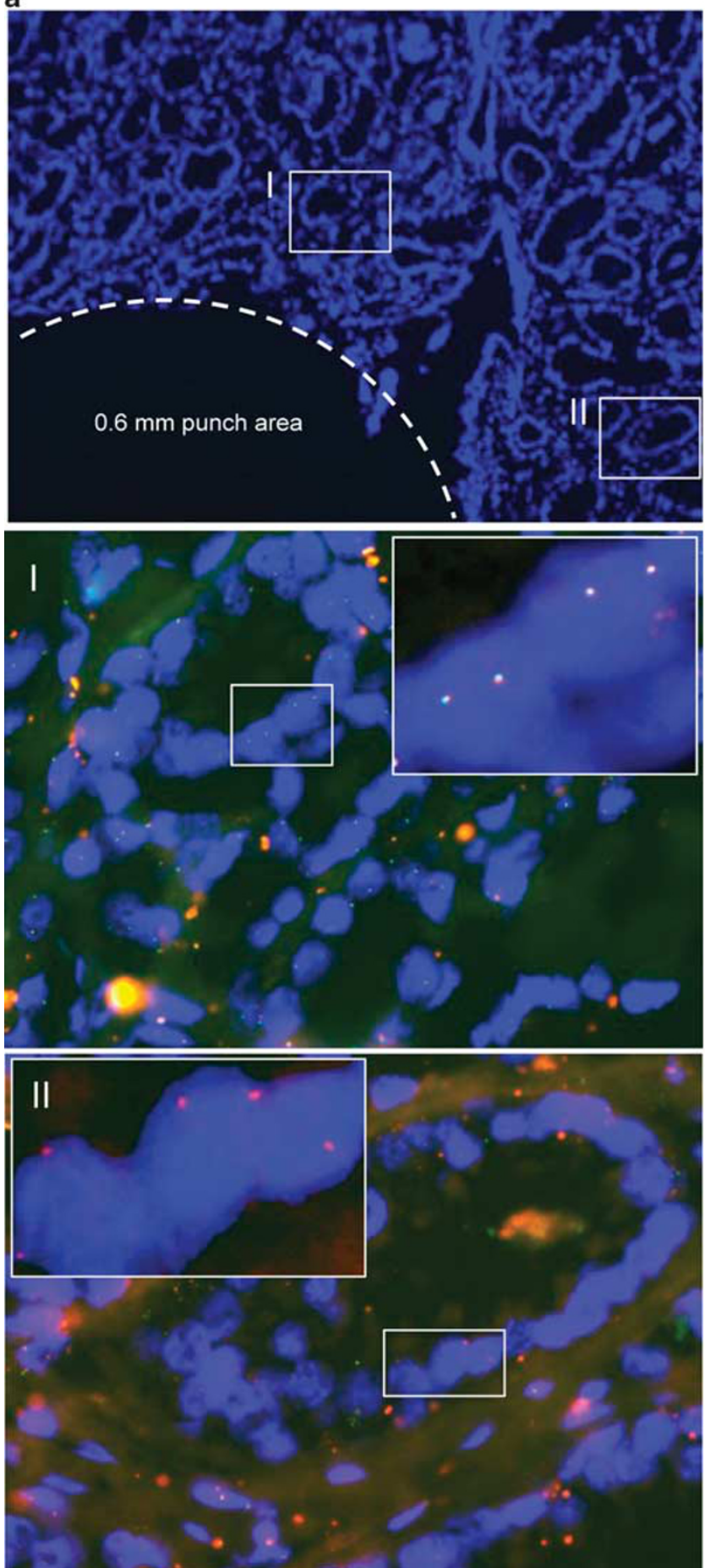

b
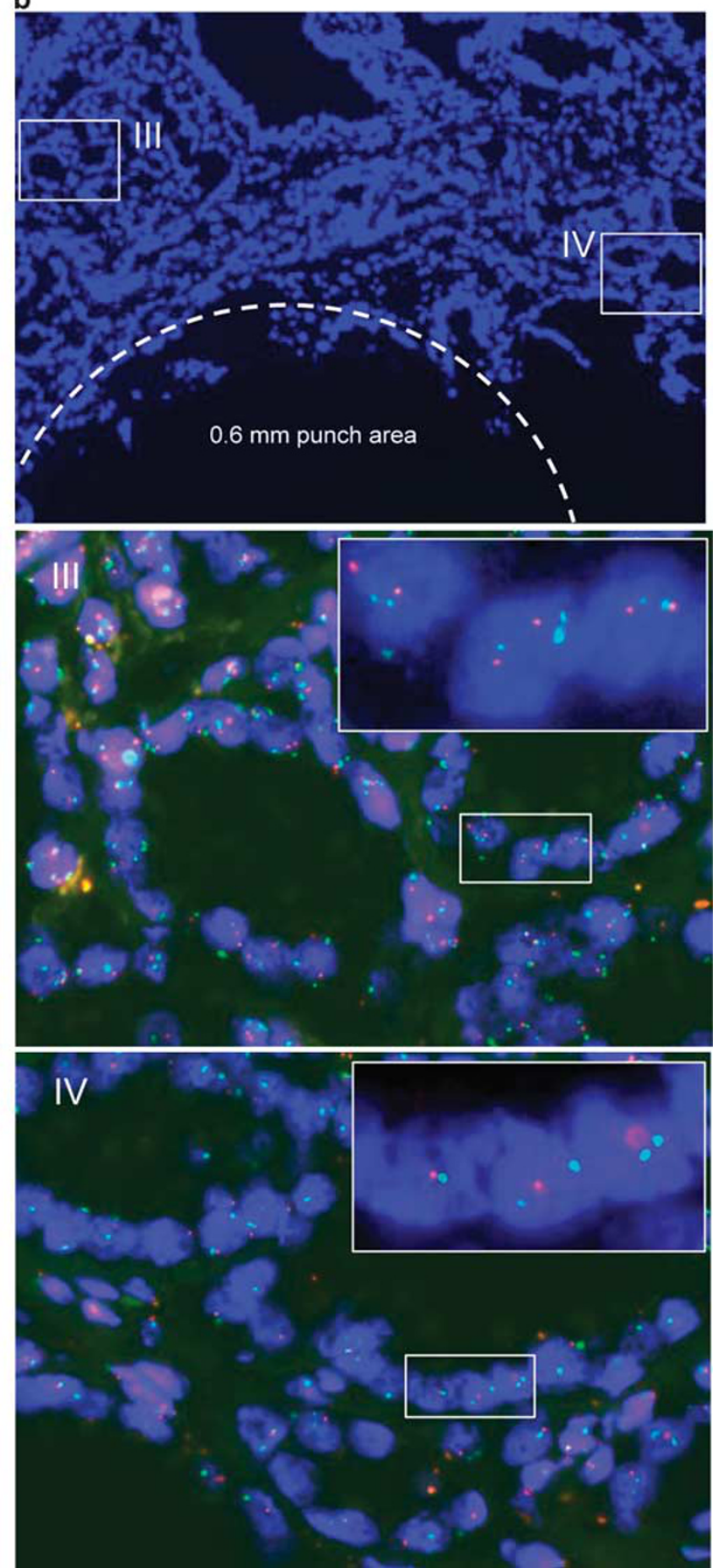

Figure 6 Examples of large section validation analysis of two cases with heterogeneous PTEN alterations. (a) Case with PTEN breakage detected with the PTEN break-apart probe. Breakage with loss of $3^{\prime}$ PTEN sequences was found in the tissue punch area taken for tissue microarray construction. An adjacent area labeled by (I) contained tumor cells with an intact PTEN locus, while another area (II) showed homozygous breakage as indicated by the loss of green signals. (b) Case with PTEN deletion detected with the deletion probe. Heterozygous PTEN deletion was found in the tissue punch area taken for tissue microarray construction. An adjacent area labeled by (III) contained tumor cells with normal PTEN locus, while another area (IV) showed heterozygous deletion indicated by loss of one red signal.

including deletions and mutations, within a single prostate cancer tumor focus ${ }^{11,17}$ and in different metastasis of a tumor. ${ }^{18}$ PTEN alterations were strongly linked to ERG fusion-positive tumor foci in our tumor set, which is in concordance with early studies by others ${ }^{8,9,19}$ and ourselves. ${ }^{10}$ 
In line with the marked heterogeneity, the overall frequency of PTEN deletions detected in our present study (48/123, 39\%) analyzing 3-10 different tissue spots per tumor was significantly higher than in our previous study analyzing only a single $0.6 \mathrm{~mm}$ tissue microarray spot per cancer (457/2266, 20\%; $P<0.0001) .{ }^{10}$ This discrepancy is explained by the higher likelihood for detecting deletions in heterogeneous tumors if multiple samples are analyzed. Along with others, , $3,20,21$ we $^{10}$ have previously shown that PTEN loss or mutation is strongly linked to aggressive and rapidly progressing prostate cancers and early tumor recurrence, making PTEN a potentially promising prognostic marker. However, the high degree of heterogeneity detected in our study indicates a significant problem for potential prognostic tests.

Next-generation sequencing studies performed by us $^{16}$ and others ${ }^{22}$ revealed that PTEN can be disrupted by chromosomal breakage, resulting in partial deletions, inversions or translocations. We have previously used a break-apart FISH probe to demonstrate presence of PTEN breakage in $4 \%$ (including cases with simultaneous deletion of the remaining allele) of prostate cancers using a large prostate cancer prognosis tissue microarray containing a single tissue microarray core per donor tumor. ${ }^{16}$ The results of our current heterogeneity study suggest that PTEN breakage may be much more frequent $(18 \%)$, but may be a transient event during the development of complete PTEN loss. This assumption is based on the observation that all tumor foci with PTEN rearrangements also had tissue spots with partial and/or complete deletion of the broken PTEN allele, which can only be explained by initial PTEN breakage and subsequent deletion of chromosomal material flanking the breakpoint. Importantly, such additional deletions occurred even in cases where PTEN was already completely inactivated, for example, by deletion of one allele and breakage of the other. This finding suggests additional selection pressure acting beyond sole inactivation of PTEN. It is possible, that the nearby Killin gene (KLLN), encoding a p53-dependent inducer of apoptosis ${ }^{23}$ drives these additional deletions. The small (537 bp) KLLN locus is located directly adjacent to the $5^{\prime}$ edge of the PTEN locus ${ }^{23}$ and, therefore, included in 10q23 deletions detected by 'typical' PTEN deletion probes including ours (Figure 1a). ${ }^{10}$ It is tempting to speculate that tumors with co-deletion of PTEN and KLLN may escape the p53-dependent fail-safe mechanism resulting in growth arrest and senescence in PTEN-deficient cells. ${ }^{24}$ Such a co-deletion model would also be compatible with earlier reports demonstrating accumulation of tumor cells with homozygous deletion during tumor progression. ${ }^{21}$

Although cancer heterogeneity limits the applicability of diagnostic, prognostic and predictive test assays, it can be useful to monitor the sequel of two or more molecular events occurring during tumor progression. Depending on the sequel of development of PTEN alterations and ERG fusion, one can expect a small area of cancer having both alterations within a larger area having only one (the earlier) of these changes. We found small PTEN altered areas in 16 of 19 homogeneously ERG-positive tumors, but we failed to see any $E R G$-positive areas within 10 $P T E N$-deficient cancer foci, suggesting that ERG fusion typically precedes alterations of PTEN. These findings would be consistent with $E R G$ fusions facilitating the development of PTEN alterations rather than a sole cooperative effect that had been earlier suggested from mouse models with prostatetargeted ERG expression and PTEN depletion, ${ }^{6,7}$ In a recent study, Rickman et $a l^{25}$ demonstrated that $E R G$ expression is associated with broad changes in chromatin topology, giving rise to specific genomic rearrangements driven by a particularly high density of ERG-binding sites located close to the breakpoints. It may be speculated that also the PTEN locus may be prone to such $E R G$-driven rearrangements.

In summary, the heterogeneity tissue microarray approach used in this study did not only allow estimating the degree of heterogeneity of individual markers, but also provided insights into the sequel of PTEN and ERG alterations in prostate cancer. The results of our study show a marked degree of heterogeneity of PTEN alterations, including deletions and gene breakage, and outline a stepwise molecular progression of PTEN alterations from heterozygous breakage to homozygous deletion. In addition, our data strongly suggest a driving role of ERG fusion to promote development of PTEN alterations.

\section{Acknowledgments}

This work was supported by the German Cancer Aid (Grant \#109505) and Sander Foundation 2012-023.1. The skillful technical assistance of Bianca Kelp, Silvia Schnoeger and Sascha Eghtessadi is gratefully acknowledged.

\section{Disclosure/conflict of interest}

The authors declare no conflict of interest.

\section{Author contributions}

AK, FF, SH, MK, SF and LB performed and analyzed the tissue microarray and large section FISH experiments; PS, MCT, MB and SS reviewed all tissue samples, determined tumor foci and selected tumors for molecular analyses, PT, MG and TS provided cancer specimens; and AK, HS, TS, GS, RS and SM interpreted all data and wrote the manuscript. All contributors were involved in approving the final version of the manuscript before submission. 


\section{References}

1 Jemal A, Siegel R, Xu J, et al. Cancer statistics, 2010. CA Cancer J Clin 2010;60:277-300.

2 Minner S, Gartner M, Freudenthaler F, et al. Marked heterogeneity of ERG expression in large primary prostate cancers. Mod Pathol 2013;26:106-116.

3 Yoshimoto M, Cunha IW, Coudry RA, et al. FISH analysis of 107 prostate cancers shows that PTEN genomic deletion is associated with poor clinical outcome. Br J Cancer 2007;97:678-685.

4 McCall P, Witton CJ, Grimsley S, et al. Is PTEN loss associated with clinical outcome measures in human prostate cancer? Br J Cancer 2008;99: 1296-1301.

5 Sircar K, Yoshimoto M, Monzon FA, et al. PTEN genomic deletion is associated with p-Akt and AR signalling in poorer outcome, hormone refractory prostate cancer. J Pathol 2009;218:505-513.

6 King JC, Xu J, Wongvipat J, et al. Cooperativity of TMPRSS2-ERG with PI3-kinase pathway activation in prostate oncogenesis. Nat Genet 2009;41:524-526.

7 Carver BS, Tran J, Gopalan A, et al. Aberrant ERG expression cooperates with loss of PTEN to promote cancer progression in the prostate. Nat Genet 2009;41: 619-624.

8 Yoshimoto M, Joshua AM, Cunha IW, et al. Absence of TMPRSS2:ERG fusions and PTEN losses in prostate cancer is associated with a favorable outcome. Mod Pathol 2008;21:1451-1460.

9 Han B, Mehra R, Lonigro RJ, et al. Fluorescence in situ hybridization study shows association of PTEN deletion with ERG rearrangement during prostate cancer progression. Mod Pathol 2009;22:1083-1093.

10 Krohn A, Diedler T, Burkhardt L, et al. Genomic deletion of PTEN is associated with tumor progression and early PSA recurrence in ERG fusion-positive and fusion-negative prostate cancer. Am J Pathol 2012;181: 401-412.

11 Reid AH, Attard G, Brewer D, et al. Novel, gross chromosomal alterations involving PTEN cooperate with allelic loss in prostate cancer. Mod Pathol 2012; 25:902-910.

12 Bismar TA, Yoshimoto M, Vollmer RT, et al. PTEN genomic deletion is an early event associated with ERG gene rearrangements in prostate cancer. BJU Int 2011; 107:477-485.
13 Minner S, Enodien M, Sirma H, et al. ERG status is unrelated to PSA recurrence in radically operated prostate cancer in the absence of antihormonal therapy. Clin Cancer Res 2011;17:5878-5888.

14 Wise AM, Stamey TA, McNeal JE, et al. Morphologic and clinical significance of multifocal prostate cancers in radical prostatectomy specimens. Urology 2002;60:264-269.

15 Minner S, Gartner M, Freudenthaler F, et al. Marked heterogeneity of ERG expression in large primary prostate cancers. Mod Pathol 2012;26:106-116.

16 Weischenfeldt J, Simon R, Feuerbach L, et al. Integrative genomic analyses reveal androgen-driven somatic alteration landscape in early-onset prostate cancer. Cancer Cell 2013;23:159-170.

17 Gerlinger M, Rowan AJ, Horswell S, et al. Intratumor heterogeneity and branched evolution revealed by multiregion sequencing. N Engl J Med 366:883-892.

18 Suzuki H, Freije D, Nusskern DR, et al. Interfocal heterogeneity of PTEN/MMAC1 gene alterations in multiple metastatic prostate cancer tissues. Cancer Res 1998;58:204-209.

19 Demichelis F, Setlur SR, Beroukhim R, et al. Distinct genomic aberrations associated with ERG rearranged prostate cancer. Genes Chromosomes Cancer 2009;48:366-380.

20 Gray IC, Stewart LM, Phillips SM, et al. Mutation and expression analysis of the putative prostate tumour-suppressor gene PTEN. Br J Cancer 1998;78: 1296-1300.

21 Verhagen PC, van Duijn PW, Hermans KG, et al. The PTEN gene in locally progressive prostate cancer is preferentially inactivated by bi-allelic gene deletion. J Pathol 2006;208:699-707.

22 Berger MF, Lawrence MS, Demichelis F, et al. The genomic complexity of primary human prostate cancer. Nature 2011;470:214-220.

23 Cho YJ, Liang P. Killin is a p53-regulated nuclear inhibitor of DNA synthesis. Proc Natl Acad Sci USA 2008;105:5396-5401.

24 Chen Z, Trotman LC, Shaffer D, et al. Crucial role of p53-dependent cellular senescence in suppression of Pten-deficient tumorigenesis. Nature 2005;436: $725-730$.

25 Rickman DS, Soong TD, Moss B, et al. Oncogenemediated alterations in chromatin conformation. Proc Natl Acad Sci USA 109:9083-9088. 